\title{
Taxonomic Notes on the Species of Hemerobiidae (Neuroptera) of Korea
}

\author{
Seulki Kim and Soowon Cho* \\ Dept of Plant Medicine, Chungbuk National University, Cheongju, CB 361-763 Korea
}

\section{국내 뱀잠자리붙이개풀잠자리목 종들의 분류학적 논고}

\author{
김슬기· 조수원* \\ 충북대학교 식물의학과
}

\begin{abstract}
Micromus variegatus (Neuroptera: Hemerobiidae) is reported here as new to South Korea. In addition, three other hemerobiid species recorded only the collection data, Hemerobius harmandinus, Sympherobius tessellatus and S. domesticus are also reported with a brief description, images of the adult and wings, and its Korean common name. With four previously reported species in Korea, there are eight species of the family Hemerobiidae from Korea.
\end{abstract}

Key words: Korean fauna, brown lacewings, Micromus variegatus

초 록: Micromus variegatus (풀잠자리목: 뱀잠자리붙이과)를 국내 미기록종으로 보고한다. 이와 함께 국외에 채집기록만 보고된 Hemerobius harmandinus, Sympherobius tessellatus 및 S. domesticus, 에 대한 간략한 기재, 성충 및 날개의 사진, 그리고 국명을 제시한다. 이로써 과거 보고 된 4 종과 함께 국내 뱀잠자리붙이과는 모두 8종으로 기록된다.

검색어: 한반도 곤충상, 뱀잠자리, Micromus variegatus, Hemerobius, Sympherobius

Hemerobiidae (brown lacewings) constitute one of the largest families of Neuroptera.

At present the family comprises more than 550 known species belonging to 43 valid genera (Monserrat, 1993; 2002). Hemerobiids are medium-sized insects, with the wings held roof-like at rest, similar to chrysopids, and generally have pale brown, frequently cryptic, coloration. The species in the family are economically interesting as biological control agents in agriculture as they are often predators of phytophagous insects such as aphids (Monserrat, 2002; Sato and Takada, 2004). Currently ten subfamilies are recognized including Adelphohemerobiinae, most recently added basal lineage (Oswald, 1993; 1994).

While over 45 species of Hemerobiidae have been reported in Japan (Tadauchi and Inoue, 2000), study of the Korean

*Comesponding author: soowon@chungbuk.ac.kr

Received February 14 2011; Revised March 8 2011;

Accepted March 92011
Hemerobiidae has been so scarce. Until recently, only the names of three species in two genera, Eumicromus numerosus (Navás, 1910), Hemerobius humulinus Linnaeus, 1758, and Ninga deltoides (Navás, 1910) have been reported new to Korea (Kwon and Moon, 1994). Of these, E. numerosus has been transferred back to Micromus numerosus Navás, 1910 and $N$. deltoides has been synonymized to Neuronema albostigma (Matsumura, 1907) (Monserrat, 2000).

Recently Micromus angulatus (Stephens, 1836) was reported as new to Korea (Kim et al., 2010). However, in a strict sense, this should be new to South Korea as it was already recorded in North Korea. In fact, recent data additions from North Korea have been reported for more than 10 species of Hemerobiidae (Monserrat, 1993; 2000). In many cases, we find that species occurring in South Korea also occur in North Korea. For example, Hemerobius harmandinus and Sympherobius tessellatus, both reported here, were also, though locality data only, reported in North Korea and 
South Korea (Miura et al., 1986). However, we also know that this is not always true as, for example, S. domesticus, another species reported here, occurs only in South Korea and Japan, but not in North Korea so far.

All the species of these examples are, however, only locality data additions, and neither descriptions, images, nor Korean common names of the species were reported. Here we report four species, $M$. variegatus, $H$. harmandinus, $S$. domesticus, and $S$. tessellatus of Hemerobiidae, with brief descriptions and images of the adults and wings, and their Korean common names. The specimens examined are deposited in the Insect Collections of the Department of Plant Medicine, Chungbuk National University in Korea (CBNU).

\section{Systematic Accounts}

\section{Genus Micromus Rambur}

Micromus Rambur, 1842:416(Type species: Hemerobius variegatus Fabricius, 1793:85, subsequently designated by Banks, 1905: 44).

\section{Micromus variegatus (Fabricius, 1793) 점박이뱀잠자 리붙이(신칭)}

Korean name: jeom-bak-i-baem-jam-ja-ri-but-i

(Figs. 1, 5)

Hemerobius variegatus Fabricius, 1793:85. (Type: syntype(s), TD and TL: unknown.).

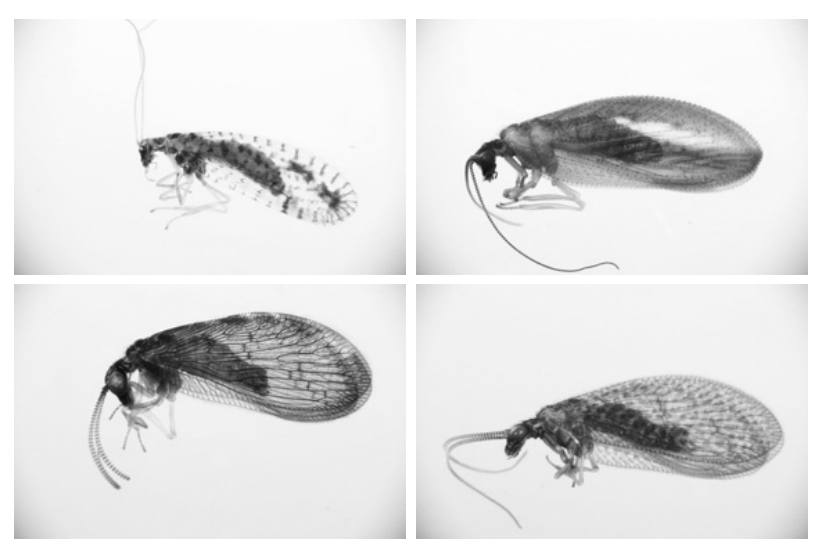

Figs. 1-4. Adults. 1, [upper left] Micromus variegatus, body length $4 \mathrm{~mm} ; 2$, [upper right] Hemerobius harmandinus, body length 5 $\mathrm{mm} ; 3$, [lower left] Sympherobius domesticus, body length $4 \mathrm{~mm}$; 4, [lower right] Sympherobius tessellatus, body length $4 \mathrm{~mm}$.
Micromus variegatus Rambur, 1842:417.

Micromus pulchellus Nakahara, 1915:33.

Diagnosis. Body length 4-5 mm, forewing 5-6 mm, hindwing 4-5 mm. Head light brown; vertex much darker; palpi light brown. Prothorax mostly dark but marginally white; meso- and metathorax light to dark brown, partially whitish. Forewing mostly white with some black spots around radius and median cross veins.

Material examined. $1 \sigma^{x}$ : CBNU, Cheongju, coll. S. K. Kim,

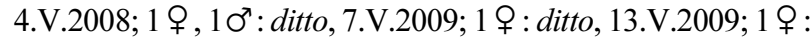
ditto, 10.VI.2008; 1오 : ditto, 12.VI.2009; 1 우: ditto, 12.VII.2007; 2 우 우: Weol-ag-san [Mt.], Chungju, coll. S. K.

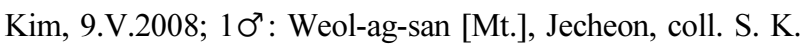
Kim, 19.IX.2009.

Distribution. Palearctic including Korea (North and South) (widespread), southwestern Canada.

Remark. The species is known to be a predator of pea aphids, Acyrthosiphon pisum (완두수염진딧물) (Dunn, 1954).

\section{Genus Hemerobius Linnaeus}

Hemerobius Linnaeus, 1758:549 (Type species: Hemerobius humulinus Linnaeus, 1758:550, subsequently designated by the International Commission on Zoological Nomenclature, Opinion 211:3).

Hemerobius harmandinus Navás, [1910] 유리날개뱀잠자리붙이(신칭)

Korean name: yu-ri-nal-gae-baem-jam-ja-ri-but-i

(Figs. 2, 6)

Hemerobius harmandinus Navás, 1909 [1910]:395. (Type: syntype(s); TD: Museum National d'Histoire Naturelle, Paris, France; TL: Kanto, Japan).

Hemerobius nitidulus, Nakahara (not Fabricius), 1915:32.

Hemerobius nakaharinus Navás, 1916:235.

Hemerobius divisus Navás, 1931:3.

Hernerobius lacunaris Navás, 1936:51.

Diagnosis. Body length 5-6 mm, forewing 8-9 $\mathrm{mm}$, hindwing 7-8 mm. Head bright black, vertex and palpi fuscous; head 
laterally very fuscous; antenna rusty. Prothorax fuscous, front and back with numerous straight hairs laterally; Mesothorax and metanotum bright rust-colored. Tibia ventrally with spindle-like small setae; tarsus, first tarsomere very long; claw elongate, bent. Forewing and hindwing somewhat slender; basal area of wing mostly fuscous, outwardly dark brown, inwardly lighter. Abdomen covered with fuscous hair.

Material examined. $2 \sigma^{\top} \sigma^{x}$ : CBNU, Cheongju, coll. S. K. Kim,

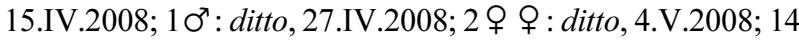
우 오, $3 \sigma^{7} \sigma^{x}$ : ditto, 7.V.2009; 10 우 우, $3 \sigma^{x} \sigma^{7}$ : ditto, 9.V.2009; 1 오 : CBNU, Cheongju, coll. G. S. Choi, 12.V.2009; 1 우 CBNU, Cheongju, coll. S. K. Kim, 28.VIII.2008; $10^{7}$ : ditto, 29.VIII.2008; $10^{7}$ : ditto, 4.IX.2008; $10^{7}, 1$ 우: Weol-ag-san [Mt.], Chungju, coll. S. K. Kim, 9.V.2008; 2 우 우, $10^{\text {t: }}$ Weol-ag-san [Mt.], Jecheon, coll. S. K. Kim, 9.V.2008.

Distribution. Eastern and southeastern Asia including Korea (North and South), Japan, China and Nepal (widespread).

Remark. Several specimen records were previously reported from Taechon[Daecheon] (Poryong[Boryeong]-gun, CN[Chungnam Province]), Pibong[Bibong] (Chongyang[Cheongyang]-gun, $\mathrm{CN}$ ), and Hongchon[Hongcheon] (Hongchon[Hongcheon]-gun, KW[GW, Gangwon Province]) in 1984 (Miura et al., 1986).

\section{Genus Sympherobius Banks}

Sympherobius Banks, 1904:209 (Type species: Hemerobius amiculus Fitch, [1855] 1854:799, by monotypy).

\section{Sympherobius domesticus Nakahara, 1954 검정뱀 잠자리붙이(신칭)}

Korean name: geom-jeong-baem-jam-ja-ri-but-i

(Figs. 3, 7)

Sympherobius domesticus Nakahara, 1954 (Type: holotype, male; TL: Mejiro, Tokyo, Japan; TD: unknown).

Diagnosis. Body length 3-4 mm, forewing 3-5 mm, hindwing 3-4 mm. Head and palpus black to fuscous black; antenna testaceous, darker toward both ends, the two basal joints black. Thorax fuscous black. Legs all pale, testaceous, tarsal joints tinged with fuscous. Forewing with very faint smoky mottling all over, hindwing hyaline. Abdomen fuscous.

Material examined. $1 \sigma^{x}$ : CBNU, Cheongju, coll. S. K. Kim, 22.V.2008; $10^{\top}$ : ditto, 4.VII.2009; 1 우 : ditto, 12.VII.2007; 1 우 : ditto, 1.VIII.2008; 3 우 우: ditto, 21.VIII.2007; 1 우: ditto, 24.VIII.2008; 10', 1 우: ditto, 27.VIII.2008; 1 우: ditto,

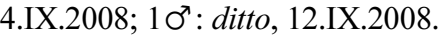

Distribution. Japan, South Korea.

Remarks. The species is known to refuse to eat aphids, but eat Phenacoccus pergandei Cockerell, the elongate cottony scales and their eggs in apple orchards (Nakahara, 1954; Kuwayama, 1962). Monserrat (2000) added collection locality data for the species in which one male and two females from Suweon[Suwon] were collected in 1974 by P. E. S. Whalley (NHM).

\section{Sympherobius tessellatus Nakahara, 1915 점날개뱀 잠자리붙이(신칭)}

Korean name: jeom-nal-gae-baem-jam-ja-ri-but-i

(Figs. 4, 8)

Sympherobius tessellatus Nakahara, 1915:22 (Type: unknown).

Sympherobius okinawensis Kuwayama, 1964:39.

Sympherobius matsucocciphagus Yang, 1980:88.

Sympherobius weisong Yang, 1980:89.

Sympherobius luojiaensis Yang, 1980:90.

Diagnosis. Body length 3-4 mm, forewing 4-5 mm, hindwing 3-4 mm. Head fuscous or fuscous black; antennae fuscous, suffused with pale yellow beyond middle ; basal joint enlarged and marked with piceous; labial palpus and maxillary palpus blackish except pale apical joint. Forewing hyaline, checkered appearance by a large number of brown spots distributed all over; hindwing hyaline; no mark except light brown pterostigmatic region. Abdomen fuscous grey.

Material examined. $10^{x}$ : CBNU, Cheongju, coll. S. K. Kim, May. 4, 2008; $10^{x}:$ ditto, May. 7, 2009; $10^{x}, 1$ 우 : ditto, May. 9, 2009; 2 우 우: ditto, Jun. 10, 2008. 


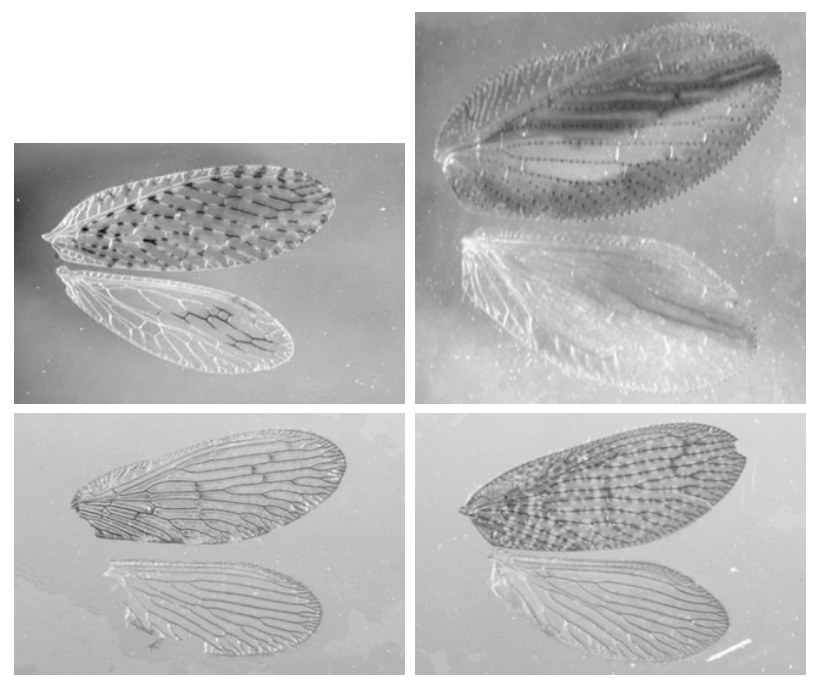

Figs. 5-8. Adult wings. 5, [upper left] Micromus variegatus, forewing length $5 \mathrm{~mm} ; 6$, [upper right] Hemerobius harmandinus, forewing length $8 \mathrm{~mm} ; 7$, [lower left] Sympherobius domesticus, forewing length $5 \mathrm{~mm} ; 8$, [lower right] Sympherobius tessellatus, forewing length $5 \mathrm{~mm}$.

Distribution. Korea (North and South), China, Japan.

Remarks. One specimen record was previously reported from Hwasong[Hwaseong], Chongyang[Cheongyang]-gun, $\mathrm{CN}$ in 1984 (Miura et al., 1986). Monserrat (2000) considered all the three new species (S. matsucocciphagus, S. weisong and $S$. luojiaensis) described by Yang (1980) did not possess any significant differences, especially on genitalia, from $S$. tessellatus and subsequently synonymized all of them. However, Yang's description such as $S$. luojiaensis having three radial sectors instead of two seems to hold a further investigation.

\section{Acknowledgements}

This work was supported by a research grant from the Chungbuk National University in 2010.

\section{Literature Cited}

Banks, N. 1904. A list of neuropteroid insects, exclusive of Odonata, from the vicinity of Washington, D.C. P. Entomol. Soc. Wash. 6: 201-217.

Banks, N. 1905. A revision of the Nearctic Hemerobiidae. T. Am. Entomol. Soc. 32: 21-51.

Dunn, J.A. 1954. Micromus variegatus Fabricius (Neuroptera) as a predator of the Pea Aphid. Proc. R. Entomol. Soc. A 29: 76-80.

Fabricius, J.C. 1793. Entomologia systematica emendata et aucta secundum classes, ordines, genera, species adjectis synonimis, locis observationibus, descriptionibus. Hafniae. Tome 2. 519 pp.

International Commission on Zoological Nomenclature. 1954. Opinion 211: Designation under the plenary powers of a type species for the genera "Hemerobius" Linnaeus, 1758, and "Chrysopa" Leach, 1815 (Class Insecta, Order Neuroptera) in harmony with accustomed usage. Opin. Decl. Int. Comm. 2001. Nom. 4: 1-14.

Kim, T.H., S.H. Oh, Y.H. Kim and J.C. Jeong. 2010. A New Record of Micromus angulatus (Neuroptera: Hemerobiidae) from Korea. Kor. J. Syst. Zool. 26(2): 157-159.

Kuwayama, S. 1962. A revisional synopsis of the Neuroptera in Japan. Pac. Insects 4: 325-412.

Kuwayama, S. 1964. On the Neuroptera of the Ryukyus. Insecta Matsumurana 27: 38-48.

Linnaeus, C. 1758. Systema natura per regna tria naturae secundum classes, ordines, genera, species, cum characteribus, differentiis, synonymis, locis. 10th Ed. Vol. 1. Salvii, Holmiae. 824 pp.

Kwon, Y.J. and T.Y. Moon. 1994. Order 22. Neuroptera. pp. 115-116. In Check List of Insects from Korea, eds. by Entomological Society of Korea and Kor. Society of Applied Entomology. 744 pp. Kon-Kuk University Press, Seoul.

Miura, T., K. Yano, M. Yasuo and B.Y. Lee. 1986. Selected insects in pine forests infested by the pine gall midge in Korea. Bull. Fac. Agr. Shimane Univ. 20: 176-190.

Monserrat, V.J. 1993. New data on some species of the genus Micromus Rambur, 1842 (Insecta: Neuroptera: Hemerobiidae). Ann. Mus. Civ. St. Nat. 89: 477-516.

Monserrat, V.J. 2000. New data on the brown lacewings from Asia (Neuroptera: Hemerobiidae). J. Neuropterology 3: 61-97.

Monserrat, V.J. 2002. Family Hemerobiidae. Pp. 238-251 (text), 398-418 (figs.). In A Guide to the Lacewings (Neuroptera) of Costa Rica, ed. by N.D. Penny. Proc. Calif. Acad. Sci. 53 (12): 161-457.

Nakahara, W. 1915. On the Hemerobiinae of Japan. Annot. Zool. Japon. 9: 11-48

Nakahara, W. 1954. Early stages of some Japanese Hemerobiidae including two new species. Kontyû 21: 41-46.

Navás, L. 1909 [1910]. Hémérobides nouveaux du Japon (Neuroptera). Rev. Russe Entomol. 9: 395-398.

Navás, L. 1916. Neurópteros nuevos o poco conocidos (Séptima serie). Mems. R. Acad. Cienc. Artes Barcelona (3) 12: 219-243.

Navás, L. 1931. Névroptères et insectes voisins. Chine et pays environnants. Deuxième [II] série. Notes Ent. Chin. 1 (7): 1-10.

Navás, L. 1936. Névroptères et insectes voisins. Chine et pays environnants. Neuvième [IX] série. Notes Ent. Chin. 3: 37-62, 117-132.

Oswald, J.D. 1993 Revision and cladistic analysis of the world genera of the family Hemerobiidae (Insecta: Neuroptera). J. New 
York Entomol. S. 101: 143-299.

Oswald, J.D. 1994. A new phylogenetically basal subfamily of brown lacewings from Chile (Neuroptera: Hemerobiidae). Entomol. Scand. 25: 295-302.

Rambur, [J.]P. 1842. Histoire Naturelle des Insectes, Névroptères. Librairie encyclopédique de Roret. Fain et Thunot, Paris. 534 pp. Sato, T. and H. Takada. 2004. Biological studies on three Micromus species in Japan (Neuroptera: Hemerobiidae) to evaluate their po- tential as biological control agents against aphids: 1 . Thermal effects on development and reproduction. Appl. Entomol. Zool. 39 (3): 417-425.

Tadauchi, O. and H. Inoue. 2000. On Mokuroku file based on "A Check List of Japanese Insects" on Internet. Esakia 40: 81-84.

Yang, C.K. 1980. Three new species of Sympherobius from China (Neuroptera: Hemerobiidae). Acta Agr. U. Pekinensis 6: 87-92. 